\title{
An Anxiolytic Response Exerted by $\beta 3$-Adrenoreceptor Activation: Correlation with an Enhanced Subset of Gabaergic Synaptic Responses
}

\author{
Robert W Greene*,I \\ 'Department of Psychiatry, UTSW and Dallas VA Medical Center, Dallas, TX, USA
}

Neuropsychopharmacology (2010) 35, I839-1840; doi:I0.1038/npp.2010.73

The behavioral effects of CNS active pharmacological agents are best understood in the context of the relevant circuit function and depending on how it is altered by these agents. Silberman et al (2010, this issue) report that a selective agonist of the $\beta 3$-adrenoreceptor ( $\beta 3$-AR) exerts an anxiolytic response when locally applied to the basolateral amygdyla (BLA). The anxiolytic and adrenergic effects in the amygdyla seem to be surprising, as $\beta$-adrenergic effects are usually associated with increased excitability and, as noted by the authors, increased BLA excitability has been strongly correlated with anxiogenic responses and decreased excitability or enhanced inhibition with anxiolytic responses (Davis et al, 1994). Indeed, facilitation of AMPA EPSCs by $\beta 1$ - and 2-AR activation (but not by $\beta 3$-AR activation) in BLA pyramidal neurons was confirmed by Silberman et al (2010, this issue). How does $\beta 3$-AR activation influence the rest of the BLA circuit activity?

B3-AR activation was observed rather to facilitate only GABAA-IPSCs evoked from stimulation of inhibitory neurons in the lateral paracapsular region of the amygdyla. Further, this facilitation required cAMP signaling in the post-synaptic pyramidal neurons, consistent with the Gs-coupling of the $\beta 3-\mathrm{AR}$. As the disruption of the cAMP signaling was confined to the recorded, post-synaptic BLA pyramidal cells (intracellular dialysis of Rp-cAMPS was used to block the IPSC facilitation), a post-synaptic $\beta 3$-AR mechanism that extends earlier observations of adrenergic mediated and PKA-dependent enhancement of GABAAmediated currents in isolated hippocampal dentate cells was established (Kapur and Macdonald, 1996). Although this does not rule out a pre-synaptic role, for example through a retrograde signal, the authors found no evidence for a change in pre-synaptic release based on paired-pulse ratio analysis. Thus, the anxiolytic effect of $\beta 3$-AR activation in

*Correspondence: Dr RW Greene, Department of Psychiatry, UTSW and Dallas VA Medical Center, 5323 Harry Hines Boulevard, Dallas, TX 75390-9070, USA, Tel: + I 214648 5108, Fax: + I 2146487037 , E-mail: robertw.greene@utsouthwestern.edu

Received 27 April 2010; accepted 28 April 2010
BLA may be attributed to selective facilitation of synaptic GABAA potentials originating from the lateral paracapsular region of the amygdyla.

The observed selectivity of the $\beta 3-\mathrm{AR}$ effect is both remarkable and puzzling. Silberman et al report that GABAA IPSCs evoked by lateral paracapsular stimulation are enhanced by $\beta 3$-AR activation, but neither the GABAA IPSCs of local neuron origin nor the AMPA EPSCs are affected by this same activation. As Gs activation leading to increased cAMP and PKA activation results in increased GABAA currents and AMPA receptor currents, why does not PKA activation by $\beta 3-\mathrm{AR}$ affect them? The authors suggest that selective localization of lateral paracapsular synapse and post-synaptic GABAA receptors with $\beta 3$-ARs may account for the selectivity. However, there is no evidence to support this. Does pharmacological activation of PKA (for example, by 8-bromo-cAMP) or widespread CAMP-induced increase result in facilitation of both excitatory and inhibitory PSPs? Does activation of all ARs, as the endogenous neurotransmitter might elicit, facilitate excitatory and both local and lateral paracapsular PSPs? Alternatively, the GABAA receptor subtype mediating the lateral paracapsular response may have greater sensitivity to PKA activation, but there is no evidence for or against this.

The anxiolytic behavior exerted by local BLA application of a $\beta 3-\mathrm{AR}$ agonist may serve to emphasize the importance of the lateral paracapsular activation of BLA pyramidal cell synapses to reduce anxiety-like behavior. As $\beta 3-\mathrm{AR}$ selectively enhances the GABAA synaptic currents, an anxiety-provoking circumstance might be expected to activate these synapses to allow their positive adrenergic modulation. In the absence of their activity, $\beta 3-\mathrm{AR}$ agonists would have no effect in this regard. It would be important to verify this activity, as it may help to provide a proof of principle for drugs targeted toward this receptor as noted by the authors.

\section{DISCLOSURE}

The author declares no conflict of interest. 


\section{REFERENCES}

Davis M, Rainnie D, Cassell M (1994). Neurotransmission in the rat amygdala related to fear and anxiety. Trends Neurosci 17: 208-214.

Kapur J, Macdonald RL (1996). Cyclic AMP-dependent protein kinase enhances hippocampal dentate granule cell GABAA receptor currents. J Neurophysiol 76: 2626-2634.
Silberman Y, Ariwodola OJ, Chappell AM, Yorgason JT, Weiner JL (2010). Lateral paracapsular GABAergic synapses in the basolateral amygdala contribute to the anxiolytic effects of $\beta 3$ adrenoceptor activation. Neuropsychopharmacology 35: 1886-1896. 